\title{
Violations of entropic Bell inequalities with coarse-grained quadrature measurements for continuous-variable states
}

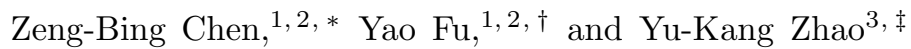 \\ ${ }^{1}$ Hefei National Laboratory for Physical Sciences at Microscale and Department of Modern Physics, \\ University of Science and Technology of China, Hefei, Anhui 230026, People's Republic of China \\ ${ }^{2}$ The CAS Center for Excellence in QIQP and the Synergetic Innovation Center for QIQP, \\ University of Science and Technology of China, Hefei, Anhui 230026, People's Republic of China \\ ${ }^{3}$ Department of Modern Physics, University of Science and Technology of China, \\ Hefei, Anhui 230026, People's Republic of China
}

(Dated: June 19, 2021)

\begin{abstract}
It is a long-standing belief, as pointed out by Bell in 1986, that it is impossible to use a twomode Gaussian state possessing a positive-definite Wigner function to demonstrate nonlocality as the Wigner function itself provides a local hidden-variable model. In particular, when one performs continuous-variable (CV) quadrature measurements upon a routinely generated CV entanglement, namely, the two-mode squeezed vacuum (TMSV) state, the resulting Wigner function is positivedefinite and as such, the TMSV state cannot violate any Bell inequality using CV quadrature measurements. We show here, however, that a Bell inequality for CV states in terms of entropies can be quantum mechanically violated by the TMSV state with two coarse-grained quadrature measurements per site within experimentally accessible parameter regime. The proposed CV entropic Bell inequality is advantageous for an experimental test, especially for a possible loophole-free test of nonlocality, as the quadrature measurements can be implemented with homodyne detections of nearly $100 \%$ detection efficiency under current technology.
\end{abstract}

PACS numbers: 03.65.Ud, 03.65.Ta, 03.67.-a, 42.50.Xa

\section{INTRODUCTION}

Local realism, as first introduced by Einstein, Podolsky and Rosen (EPR) in their famous paper [1], is the cornerstone of one's classical intuitions, namely, physical systems have local "elements of reality" no matter which experiment actually was performed. Then, the most radical departure of quantum mechanics from the classical intuitions is the discovery of Bell's inequalities [2, 3], which enable quantitative tests of quantum mechanics against local realism. While the original Bell inequalities (or in a broader sense, Bell's theorem) were derived for discrete quantum variables, their various extensions [4] have been developed for a large number of different settings. Particularly, the original EPR paradox for continuous variables (CVs) has been a source of renewed interest for topics such as the preparation of the EPR-type states [5 9] and nonlocality [10 18].

For a two-mode Gaussian state possessing a positivedefinite Wigner function, the Wigner function itself provides a local hidden-variable model, and thus it is impossible to use the state to demonstrate nonlocality. This is a long-standing belief pointed out by Bell in 1986 [15, 19]. A particular example is the two-mode squeezed vacuum (TMSV) state [5- [9], which is a routinely generated CV entanglement source and useful for various CV

\footnotetext{
*Electronic address: zbchen@ustc.edu.cn

${ }^{\dagger}$ Electronic address: yaofu@mail.ustc.edu.cn

‡Electronic address: ykzhao@mail.ustc.edu.cn
}

quantum information tasks [8]. The Wigner function of the TMSV state with CV quadrature measurements is positive-definite. Therefore, the TMSV state cannot violate any Bell inequality under the quadrature measurement settings. For this reason, the existing proposals for the Bell test with CV systems [10 18] make use of correlation functions for the parity operators [10, 11], the "parity-spin" operators [13], or the quantities acquired by a binning process to convert the continuous outcomes into binary results $14-16$, enabling us to use the ordinary form of Bell's inequalities. To take the full capacity of $\mathrm{CV}$ nature, other strategies were proposed with additional mechanisms, like using non-Gaussian states together with certain (effective) nonlinearity, but with relatively small violations [15, 17], or more involved modes [18].

We show in this paper, however, that a Bell inequality for CV states in terms of entropies can be quantum mechanically violated by the TMSV state with two coarsegrained quadrature measurements per site using the homodyne detection technique. Bell's inequalities formulated in an information-theoretic context 20 22], i.e., the entropic Bell inequalities, were first proposed by Braunstein and Caves and present a new angle to the conceptually important topic, namely, the quantum violations of local realism. An appealing feature of the entropic Bell inequalities is that they are applicable to a pair of $N$-level quantum systems for arbitrary $N$. Despite this, it is still a nontrivial question on how to demonstrate the quantum violations of local realism with $\mathrm{CV}$ systems, using information-theoretic Bell inequalities. For the very definition of information or entropy, there are important dif- 
ferences [23] between the CV and discrete-variable cases. To circumvent the difficulty caused by these differences, the proposed $\mathrm{CV}$ entropic Bell inequality uses a pair of coarse-grained quadrature measurements per site. In this way, only experimentally measured discrete probability distributions [24, 25] are involved. Furthermore, instead of correlation functions as used in the usual Bell inequalities, we use the conditional or mutual entropies to give the constraints of local realism. We then demonstrate the quantum mechanical violations of the $\mathrm{CV}$ entropic Bell inequality by the TMSV state without any use of non-Gaussian states or more involved modes. The CV entropic Bell inequality as proposed here is friendly to an experimental test, especially for a possible loopholefree test of nonlocality, as the homodyne detections have nearly 100\% detection efficiency [26] under current technology.

\section{THE CV ENTROPIC BELL INEQUALITY}

An important trick in our argument is that, instead of using probability density $p(a)$ for a continuous random variable a [23], we use only experimentally measured discrete probability distributions [24], which were proved to be very useful for witnessing CV entanglement [25]. As the continuous probability density $p(a)$ cannot be determined with a finite number of measurements, one can, however, measure a to discrete windows $\mathbf{A}_{\ell}$ of size $\Delta a$ (coarse-grained measurements). Namely, the continuous random variable $\mathbf{a}$ is discretized into equally spaced windows of size $\Delta a$ reflecting the precision of the experimental setup. Then the probability of measuring a to be in window $\mathbf{A}_{\ell}$ reads [24, 25]

$$
P\left(\mathbf{A}_{\ell}\right) \equiv \int_{\Delta a_{\ell}} \operatorname{dap}(a)
$$

where the integration is performed over $\Delta a_{\ell} \equiv\left[a_{\ell}-\right.$ $\left.\frac{1}{2} \Delta a, a_{\ell}+\frac{1}{2} \Delta a\right]$ with $a_{\ell}=\ell \cdot \Delta a(\ell=0, \pm 1, \pm 2, \ldots)$. The Shannon entropy of this discrete probability distribution $P\left(\mathbf{A}_{\ell}\right)$ is given by

$$
S(\mathbf{A})=-\sum_{\ell} P\left(\mathbf{A}_{\ell}\right) \ln P\left(\mathbf{A}_{\ell}\right) .
$$

The usual differential information (or entropy) for the continuous random variable a with probability density $p(a)$ reads

$$
s(\mathbf{a})=-\int d a p(a) \ln p(a) .
$$

Note that $s(\mathbf{a})$ is defined up to an arbitrary constant and can even be arbitrarily large, positive or negative, because of the continuous nature of the random variable a. This property of the CV information is in contrast to the discrete-variable cases.

For two random variables $\mathbf{a}$ and $\mathbf{b}$ having a joint probability density $p(a, b)$, we can define correspondingly an information $s(\mathbf{a}, \mathbf{b})=-\int d a d b p(a, b) \ln p(a, b)$. The conditional information reads

$$
s(\mathbf{a} \mid \mathbf{b})=-\int d b p(b) \int d a p(a \mid b) \ln p(a \mid b)
$$

in terms of the conditional probability density $p(a \mid b)$. Using Bayes' theorem, i.e., $p(a, b)=p(a \mid b) p(b)=$ $p(b \mid a) p(a)$, one has

$$
s(\mathbf{a}, \mathbf{b})=s(\mathbf{a} \mid \mathbf{b})+s(\mathbf{b})=s(\mathbf{b} \mid \mathbf{a})+s(\mathbf{a}) .
$$

Similarly, one can define the Shannon entropy $S(\mathbf{A}, \mathbf{B})$ of the discrete probability distribution $P\left(\mathbf{A}_{\ell}, \mathbf{B}_{m}\right)$. The discretized conditional entropy is then

$$
S(\mathbf{A} \mid \mathbf{B})=S(\mathbf{A}, \mathbf{B})-S(\mathbf{B}) .
$$

In terms of the above discretized entropies we have two useful inequalities

$$
S(\mathbf{A}, \mathbf{B}) \geq S(\mathbf{A}) \geq S(\mathbf{A} \mid \mathbf{B}),
$$

which have a transparent information-theoretic interpretation. The first inequality stems from the fact that the information carried by two quantities is never less than the information separately carried by either quantity. The information carried by a quantity never decreases by removing a condition. This then leads to the last inequality. Note that for the CV case, one does not have the inequalities $s(\mathbf{a}, \mathbf{b}) \geq s(\mathbf{a}) \geq s(\mathbf{a} \mid \mathbf{b})$.

To derive the required entropic Bell inequality we follow the reasoning by Braunstein and Caves [20]. Consider now two space-like separated $\mathrm{CV}$ quantum systems $A$ and $B$. For system $A(B)$ we have two measurable quantities $\mathbf{a}$ and $\mathbf{a}^{\prime}$ ( $\mathbf{b}$ and $\mathbf{b}^{\prime}$ ) whose values are denoted by continuous random variables $a$ and $a^{\prime}\left(b\right.$ and $\left.b^{\prime}\right)$. Quantum mechanically, two incompatible observables (e.g., a and $\mathbf{a}^{\prime}$ for system $A$ ) of a system cannot be measured simultaneously. Hence in each run of the two-setting Bell experiments one can only measure two observables (here, e.g., $\mathbf{a}$ and $\mathbf{b}$ ), one from each system. By contrast, local realism implies that the four quantities specified above are all local objective properties of the whole system. An important consequence of the observation is the existence of a joint probability density $p\left(a, a^{\prime}, b, b^{\prime}\right)$, from which we can obtain appropriate probability densities, e.g.,

$$
p(a, b)=\int d a^{\prime} d b^{\prime} p\left(a, a^{\prime}, b, b^{\prime}\right),
$$

the marginals of the joint probability density $p\left(a, a^{\prime}, b, b^{\prime}\right)$. Then other relevant probability densities can also be obtained, e.g., $p(a)=\int d a p(a, b)$.

With these probability densities in mind, we can define the corresponding discrete entropies, in terms of which we have the following information inequality [20]

$$
S(\mathbf{A}, \mathbf{B}) \leq S\left(\mathbf{A}, \mathbf{B}^{\prime}, \mathbf{A}^{\prime}, \mathbf{B}\right),
$$

where $S\left(\mathbf{A}, \mathbf{B}^{\prime}, \mathbf{A}^{\prime}, \mathbf{B}\right)=S\left(\mathbf{A} \mid \mathbf{B}^{\prime}, \mathbf{A}^{\prime}, \mathbf{B}\right)+$ $S\left(\mathbf{B}^{\prime} \mid \mathbf{A}^{\prime}, \mathbf{B}\right)+S\left(\mathbf{A}^{\prime} \mid \mathbf{B}\right)+S(\mathbf{B})$. Using the facts that 
$S\left(\mathbf{A} \mid \mathbf{B}^{\prime}, \mathbf{A}^{\prime}, \mathbf{B}\right) \leq S\left(\mathbf{A} \mid \mathbf{B}^{\prime}\right), S\left(\mathbf{B}^{\prime} \mid \mathbf{A}^{\prime}, \mathbf{B}\right) \leq S\left(\mathbf{B}^{\prime} \mid \mathbf{A}^{\prime}\right)$ and Eq. (6), we have the entropic Bell inequality

$$
0 \leq S\left(\mathbf{A} \mid \mathbf{B}^{\prime}\right)+S\left(\mathbf{B}^{\prime} \mid \mathbf{A}^{\prime}\right)+S\left(\mathbf{A}^{\prime} \mid \mathbf{B}\right)-S(\mathbf{A} \mid \mathbf{B}) .
$$

This is an information-theoretic constraint that has to be obeyed by all local realistic theories. Similar to Ref. 22] we can also obtain an entropic Bell inequality in terms of the mean mutual information $S\left(\mathbf{A} ; \mathbf{B}^{\prime}\right)+S\left(\mathbf{A}^{\prime} ; \mathbf{B}^{\prime}\right)+$ $S\left(\mathbf{A}^{\prime} ; \mathbf{B}\right)-S(\mathbf{A} ; \mathbf{B}) \leq S\left(\mathbf{A}^{\prime}\right)+S\left(\mathbf{B}^{\prime}\right)$, which takes a form quite similar to the Clauser-Horne-Shimony-Holt inequalities [3]. Here $S(\mathbf{A} ; \mathbf{B})=S(\mathbf{A})+S(\mathbf{B})-S(\mathbf{A}, \mathbf{B})$.

\section{QUANTUM VIOLATION OF THE CV ENTROPIC BELL INEQUALITY}

Now let us show the quantum violation of the entropic Bell inequality Eq. (10) by the regularized EPR states [57, 12] produced in a pulsed nondegenerate optical parametric amplification process. The process generates the TMSV state associated with two quantized light modes (denoted by the corresponding annihilation operators $\hat{a}$ and $\hat{b})$ as

$$
\begin{aligned}
|\mathrm{TMSV}\rangle & =e^{r\left(\hat{a}^{\dagger} \hat{b}^{\dagger}-\hat{a} \hat{b}\right)}|00\rangle=\frac{1}{\cosh r} e^{\hat{a}^{\dagger} \hat{b}^{\dagger} \tanh r}|00\rangle \\
& =\frac{1}{\cosh r} \sum_{n=0}^{\infty}(\tanh r)^{n}|n, n\rangle .
\end{aligned}
$$

Here, for simplicity, we assume that $r>0$ known as the squeezing parameter and $|n n\rangle \equiv|n\rangle_{a}|n\rangle_{b}=$ $\frac{1}{n !} \hat{a}^{\dagger n} \hat{b}^{\dagger n}|00\rangle$. In the infinite squeezing limit, the TMSV state $\mid$ TMSV $\rangle$ becomes the original, normalized EPR state [7, 13]; i.e., $|\mathrm{TMSV}\rangle \stackrel{r \rightarrow \infty}{\longrightarrow}|\mathrm{EPR}\rangle_{\text {normalized }}$. Such a CV entanglement can now be routinely generated and is a vital resource for various CV quantum information tasks [8].

For each mode of the light field, one measures the quadrature phase amplitude operators

$$
\hat{\mathbf{a}}_{\theta}=\frac{1}{\sqrt{2}}\left(\hat{a}^{\dagger} e^{i \theta}+\hat{a} e^{-i \theta}\right), \quad \hat{\mathbf{b}}_{\phi}=\frac{1}{\sqrt{2}}\left(\hat{b}^{\dagger} e^{i \phi}+\hat{b} e^{-i \phi}\right),
$$

with the usual homodyne measurement technique. Note that $\left[\hat{\mathbf{a}}_{\theta}, \hat{\mathbf{a}}_{\theta+\pi / 2}\right]=i$. Thus, $\hat{\mathbf{a}}_{\theta}$ and $\hat{\mathbf{a}}_{\theta+\pi / 2}$ (similarly for $\hat{\mathbf{b}}_{\phi}$ and $\hat{\mathbf{b}}_{\phi+\pi / 2}$ ) form a canonically conjugate pair. Experimental control of the local oscillator phases $(\theta$ and $\phi)$ provides access to the continuous distribution of the quadratures $\hat{\mathbf{a}}_{\theta}$ and $\hat{\mathbf{b}}_{\phi}$. Let us denote the eigenvectors of $\hat{\mathbf{a}}_{\theta}$ and $\hat{\mathbf{b}}_{\phi}$ by $\left|a_{\theta}\right\rangle$ and $\left|b_{\phi}\right\rangle$, respectively, namely, $\hat{\mathbf{a}}_{\theta}\left|a_{\theta}\right\rangle=a_{\theta}\left|a_{\theta}\right\rangle$ and $\hat{\mathbf{b}}_{\phi}\left|b_{\phi}\right\rangle=b_{\phi}\left|b_{\phi}\right\rangle$. Then the quantum pair probability density for measuring $\hat{\mathbf{a}}_{\theta}$ and $\hat{\mathbf{b}}_{\phi}$ (with the results $a_{\theta}$ and $b_{\phi}$ ) upon $|\mathrm{TMSV}\rangle$ can be calculated as

$$
p_{\mathrm{QM}}\left(a_{\theta}, b_{\phi}\right)=\mid\left\langle a_{\theta}\left|\left\langle b_{\phi} \mid \mathrm{TMSV}\right\rangle\right|^{2} .\right.
$$

To evaluate $p_{\mathrm{QM}}\left(a_{\theta}, b_{\phi}\right)$, we need some useful properties 27] of $\left|a_{\theta}\right\rangle$ (similarly for $\left|b_{\phi}\right\rangle$ ), namely,

$$
\left\langle a_{\theta} \mid a_{\theta}^{\prime}\right\rangle=\delta\left(a_{\theta}-a_{\theta}^{\prime}\right), \quad \int d a_{\theta}\left|a_{\theta}\right\rangle\left\langle a_{\theta}\right|=\hat{I}_{A},
$$

which are the orthogonal and completeness relations for $\left|a_{\theta}\right\rangle$, similar to the usual eigenvectors of a position operator. Here, $\hat{I}_{A}$ is the identity operator for system $A$. Moreover,

$$
\begin{aligned}
\left\langle a_{\theta} \mid n\right\rangle_{a} & =\frac{1}{\sqrt{\sqrt{\pi} 2^{n} n !}} \exp \left(-i n \theta-a_{\theta}^{2} / 2\right) H_{n}\left(a_{\theta}\right), \\
\left\langle b_{\phi} \mid n\right\rangle_{b} & =\frac{1}{\sqrt{\sqrt{\pi} 2^{n} n !}} \exp \left(-i n \phi-b_{\phi}^{2} / 2\right) H_{n}\left(b_{\phi}\right),
\end{aligned}
$$

where $H_{n}$ is the Hermite polynomial of order $n$. Using Eq. (15) we can write down $\left|a_{\theta}\right\rangle$ explicitly [27]

$$
\left|a_{\theta}\right\rangle=\pi^{-1 / 4} \exp \left[-\frac{1}{2} a_{\theta}^{2}+\sqrt{2} e^{i \theta} a_{\theta} \hat{a}^{\dagger}-\frac{1}{2} e^{2 i \theta} \hat{a}^{\dagger 2}\right]|0\rangle .
$$

Hence, one can evaluate $p_{\mathrm{QM}}\left(a_{\theta}, b_{\phi}\right)$ using either Eq. (15) or Eq. (16).

Here we calculate $p_{\mathrm{QM}}\left(a_{\theta}, b_{\phi}\right)$ with the help of Eq. (15). To begin with, we use Mehler's formula [28],

$$
\sum_{n=0}^{+\infty} \frac{H_{n}(x) H_{n}(y)}{2^{n} n !} t^{n}=\frac{1}{\sqrt{1-t^{2}}} e^{\left[2 x y t-\left(x^{2}+y^{2}\right) t^{2}\right] /\left(1-t^{2}\right)},
$$

where $|t|<1$. Consequently, using Eqs. (11) and (15) yields

$$
\left\langle a_{\theta}\right|\left\langle b_{\phi} \mid \mathrm{TMSV}\right\rangle=\frac{e^{-\left(a_{\theta}^{2}+b_{\phi}^{2}\right) / 2} e^{\left[2 a_{\theta} b_{\phi} t-\left(a_{\theta}^{2}+b_{\phi}^{2}\right) t^{2}\right] /\left(1-t^{2}\right)}}{\sqrt{\pi} \sqrt{1-t^{2}} \cosh r},
$$

with $t=e^{-i(\theta+\phi)} \tanh r$ (Note that indeed $|t|<1$ as $\tanh r<1$ for $r>0$ ). Hereafter, we take $\varphi=\theta+\phi$. Then

$$
\begin{aligned}
p_{\mathrm{QM}}\left(a_{\theta}, b_{\phi}\right) & =\frac{e^{-\left(a_{\theta}^{2}+b_{\phi}^{2}\right)}\left|e^{\left[2 a_{\theta} b_{\phi} t-\left(a_{\theta}^{2}+b_{\phi}^{2}\right) t^{2}\right] /\left(1-t^{2}\right)}\right|^{2}}{\pi\left|1-t^{2}\right| \cosh ^{2} r} \\
& =\frac{e^{-\left(a_{\theta}^{2}+b_{\phi}^{2}\right) v+2 a_{\theta} b_{\phi} w}}{\pi\left|1-t^{2}\right| \cosh ^{2} r}
\end{aligned}
$$

which shows the explicit anti-correlations between the local oscillator phases $\theta$ and $\phi$. Here $\left|1-t^{2}\right|=$ $\sqrt{1+\tanh ^{4} r-2 \tanh ^{2} r \cos 2 \varphi}$ and

$$
\begin{aligned}
v & =\frac{1-\tanh ^{4} r}{\left|1-t^{2}\right|^{2}} \\
w & =\frac{2 \tanh r \cos \varphi\left(1-\tanh ^{2} r\right)}{\left|1-t^{2}\right|^{2}} .
\end{aligned}
$$

From the Gaussian integral formula

$$
\int \exp \left(-\sum_{i, j=1}^{m} x_{i} \mathcal{A}_{i j} x_{j}\right) d^{m} x=\sqrt{\frac{\pi^{m}}{\operatorname{det} \mathcal{A}}},
$$


we obtain in particular that $\int d x d y \exp \left[-\left(x^{2}+y^{2}\right) \alpha+\right.$ $2 x y \beta]=\pi / \sqrt{\alpha^{2}-\beta^{2}} \equiv J(\alpha, \beta)$, where $\alpha>0$. Here, $\mathcal{A}_{m \times m}$ is a symmetric positive-definite (hence invertible) covariance matrix. Then we can confirm the normalization condition of $p_{\mathrm{QM}}\left(a_{\theta}, b_{\phi}\right)$,

$$
\int d a_{\theta} d b_{\phi} p_{\mathrm{QM}}\left(a_{\theta}, b_{\phi}\right)=\frac{\pi / \sqrt{v^{2}-w^{2}}}{\pi\left|1-t^{2}\right| \cosh ^{2} r}=1,
$$

as can easily be checked by using Eq. (20). From $p_{\mathrm{QM}}\left(a_{\theta}, b_{\phi}\right)$ we can easily obtain

$$
p_{\mathrm{QM}}\left(b_{\phi}\right)=\int d a_{\theta} p_{\mathrm{QM}}\left(a_{\theta}, b_{\phi}\right)=\frac{e^{-b_{\phi}^{2} / \cosh 2 r}}{\sqrt{\pi \cosh 2 r}} .
$$

Now let us calculate $S_{\mathrm{QM}}\left(\mathbf{B}_{\phi}\right)$ and $S_{\mathrm{QM}}\left(\mathbf{A}_{\theta}, \mathbf{B}_{\phi}\right)$. Hereafter, we take base of the logarithm to be $e$. By definition, $S_{\mathrm{QM}}\left(\mathbf{B}_{\phi}\right)=$ $-\sum_{m} P_{\mathrm{QM}}\left(\mathbf{B}_{\phi, m}\right) \ln P_{\mathrm{QM}}\left(\mathbf{B}_{\phi, m}\right)$ and $S_{\mathrm{QM}}\left(\mathbf{A}_{\theta}, \mathbf{B}_{\phi}\right)=$ $-\sum_{\ell, m} P_{\mathrm{QM}}\left(\mathbf{A}_{\theta, \ell}, \mathbf{B}_{\phi, m}\right) \ln P_{\mathrm{QM}}\left(\mathbf{A}_{\theta, \ell}, \mathbf{B}_{\phi, m}\right)$, where

$$
\begin{aligned}
P_{\mathrm{QM}}\left(\mathbf{B}_{\phi, m}\right) & =\int_{\Delta b_{\phi, m}} d b_{\phi} p_{\mathrm{QM}}\left(b_{\phi}\right), \\
P_{\mathrm{QM}}\left(\mathbf{A}_{\theta, \ell}, \mathbf{B}_{\phi, m}\right) & =\int_{\Delta a_{\theta, \ell}} d a_{\theta} \int_{\Delta b_{\phi, m}} d b_{\phi} p_{\mathrm{QM}}\left(a_{\theta}, b_{\phi}\right) .
\end{aligned}
$$

With these results in mind, we get the quantum discretized conditional entropy under the given measurement precisions $\left(\Delta a_{\theta}\right.$ and $\left.\Delta b_{\phi}\right)$ :

$$
S_{\mathrm{QM}}\left(\mathbf{A}_{\theta} \mid \mathbf{B}_{\phi}\right)=S_{\mathrm{QM}}\left(\mathbf{A}_{\theta}, \mathbf{B}_{\phi}\right)-S_{\mathrm{QM}}\left(\mathbf{B}_{\phi}\right) \equiv S_{\mathrm{QM}}(\varphi) .
$$

The negativity of $S_{\mathrm{QM}}\left(\mathbf{A}_{\theta} \mid \mathbf{B}_{\phi}\right)$ rules out any description of local realism, or an underlying joint probability distribution [22].

To demonstrate that the entropic Bell inequality Eq. (10) can indeed be violated quantum mechanically, we take the following angles:

$$
\phi=-\theta+\delta, \quad \theta^{\prime}=\theta-2 \delta / 3, \quad \phi^{\prime}=-\theta+\delta / 3 .
$$

Then the right-hand side of Eq. (10) becomes [Note that $\left.S_{\mathrm{QM}}(-\varphi)=S_{\mathrm{QM}}(\varphi)\right]$

$$
\mathcal{D}_{\mathrm{QM}}(r, \delta)=3 S_{\mathrm{QM}}\left(\frac{\delta}{3}\right)-S_{\mathrm{QM}}(\delta)
$$

Here, we assumed that $\Delta a_{\theta}=\Delta a_{\theta^{\prime}} \equiv \Delta a$ and $\Delta b_{\phi}=$ $\Delta b_{\phi^{\prime}} \equiv \Delta b$. The negativity of $\mathcal{D}_{\mathrm{QM}}$ implies the quantum violations of the entropic Bell inequality Eq. (10) and gives the deficit information [20] carried by systems $A$ and $B$, relative to that imposed by local realism under the same geometry.

In Fig. 1 we plot $\mathcal{D}_{\mathrm{QM}}$ as a function of $\delta$ and $r$ for different values of $\Delta a=\Delta b \equiv \Delta$. We also show the plane of $\mathcal{D}_{\mathrm{QM}}=0$, above which is the parameter regime of violating the inequality Eq. (10). Numeric result at $\Delta=1.5$ shows only tiny violation for large squeezing
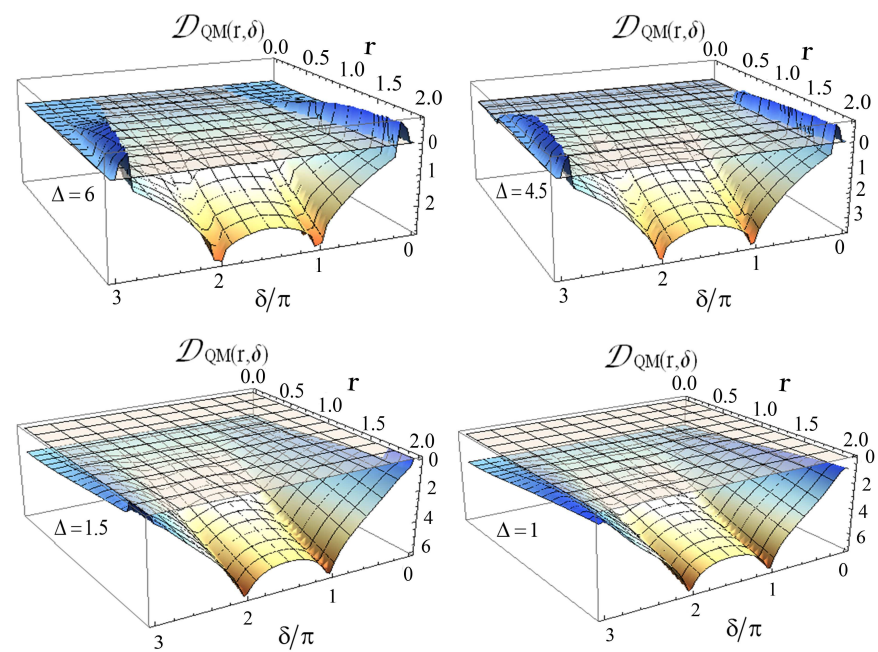

FIG. 1: (Color online). The plot of $\mathcal{D}_{\mathrm{QM}}$ [see Eq. (27)] as a function of angle $\delta$ and the squeezing parameter $r$ for different values of the measurement resolutions $\Delta$. We also show the plane of $\mathcal{D}_{\mathrm{QM}}=0$, above which is the parameter regime of violating the inequality Eq. (10). An apparent trend is that larger $\Delta$ requires smaller $r$ to violate the entropic Bell inequality.

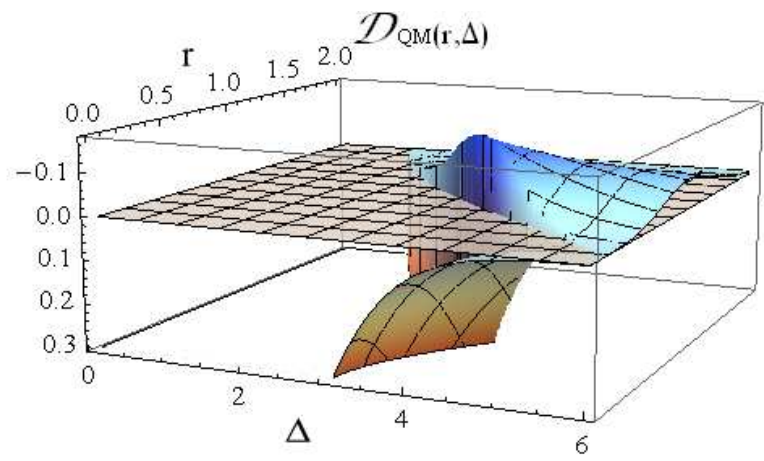

FIG. 2: (Color online). The plot of $\mathcal{D}_{\mathrm{QM}}$ at $\delta=0$ as a function of the squeezing parameter $r$ and the measurement resolution $\Delta$. The parameter regime violating the inequality Eq. (10) is above the plane of $\mathcal{D}_{\mathrm{QM}}(r, \Delta, \delta=0)=0$.

parameters in the vicinity of $r=2$, while no violation was found for $\Delta=1$ and $r \in[0,2]$. From Fig. 1, we note that a large fraction of the violations occur in the vicinity of $\delta=0$ (as well as its symmetric point $\delta=3 \pi$ ). We then plot $\mathcal{D}_{\mathrm{QM}}$ at $\delta=0$ as a function of $\Delta$ and $r$ in Fig. 2, where the plane of $\mathcal{D}_{\mathrm{QM}}(r, \Delta, \delta=0)=0$ is shown, too. In this specific case the parameter regime of violating the entropic Bell inequality indicates that larger values of $\Delta$ require smaller squeezing parameters $r$, a similar trend observed from Fig. 1. Thus, the proposed violations are experimentally more accessible for coarsegrained measurements with larger $\Delta$.

However, if we only consider the maximal violations of 
the inequality Eq. (10), namely, the minimal value $\mathcal{D}_{\mathrm{QM}}^{\mathrm{min}}$ of $\mathcal{D}_{\mathrm{QM}}(r, \Delta, \delta)$ for a given parameter regime, a complicated interplay between $r$ and $\Delta$ is observed during our numeric calculations. For example, one can detect two minimal values of $\mathcal{D}_{\mathrm{QM}}(r, \Delta, \delta)$ for the parameter regime of $r \in[0,2], \delta \in[0, \pi]$, and small $\Delta(\leq 10)$; i.e., $\mathcal{D}_{\mathrm{QM}}^{\min } \approx-0.75$ at $r \approx 1.817, \Delta=6, \delta \approx 0.213 \pi$ and $r \approx 1.915, \Delta=3.5, \delta \approx 0.098 \pi$, respectively. We also calculate $\mathcal{D}_{\mathrm{QM}}^{\min }$ for larger $\Delta$. For $\Delta=30, \mathcal{D}_{\mathrm{QM}}^{\min } \approx-0.0016$ $(r \approx 1.991, \delta \approx 0.502 \pi)$ for $r \in[0,2],-0.685(r \approx 3$, $\delta \approx 0.335 \pi)$ for $r \in[0,3],-1(r \approx 3.756, \delta \approx 0.0004 \pi)$ for $r \in[0,4]$, indicating more violations for larger $r$ with the given $\Delta$. For the parameter regime of $r \in[0,4]$ and $\delta \in[0, \pi]$ with even larger $\Delta$, we show two examples, namely, $\Delta=50, \mathcal{D}_{\mathrm{QM}}^{\min } \approx-1.9(r \approx 3.781, \delta \approx 0.0004 \pi)$, while $\mathcal{D}_{\mathrm{QM}}^{\min } \approx-0.549(r \approx 3.901, \delta \approx 0.0065 \pi)$ for $\Delta=100$. From all these numeric calculations, we see that the maximal violation of the inequality Eq. (10) is strongly influenced by the parameters of $r$ and $\Delta$, as well as their interplay. Intuitively, one might expect that our coarse-grained measurement effectively becomes the usual discrete measurements for sufficiently large $\Delta$. However, it is quite difficult to get a simple picture due to the complicated interplay between $r$ and $\Delta$ as we observed in our numeric calculations.

Finally, let us briefly discuss the remaining experimental issues regarding the test of the entropic Bell inequality Eq. (10). For given $r$ and $\delta$, the joint probability distribution $P_{\mathrm{QM}}\left(\mathbf{A}_{\theta, \ell}, \mathbf{B}_{\phi, m}\right)$ can be experimentally measured [25, 29] from the homodyne detection data, giving rise to $P_{\mathrm{QM}}\left(\mathbf{B}_{\phi, m}\right)$ as marginal probability distribution. For experimentally closing the locality loophole, it is important to use random and fast switching of the local measurement settings such that the space-time separated measurements are guaranteed. All previous Bell experiments could in principle be challenged by the detectionefficiency and/or locality loopholes [30]. The former loophole has been closed in Ref. [31] by using entangled ions, which can be detected with nearly perfect efficiency. The entangled photons are ideal for closing the locality loop- hole, as pioneered by Aspect et al. [32] and then by Weihs et al. 33. Our proposal has used the pulsed 34 CV entangled source (being easy to close the locality loophole) and the quadrature measurement with homodyne detections (having nearly unit detection efficiency), thus opening up the exciting possibility for a loophole-free test of local realism against quantum mechanics.

\section{CONCLUSIONS AND DISCUSSIONS}

In summary, an entropic Bell inequality has been proposed for $\mathrm{CV}$ states. Our argument requires simply coarse-grained quadrature measurements per site, without any need of experimentally complicated mechanisms such as non-Gaussian states and nonlinearity [15, 17] or more involved modes [18]. Thus, this is the simplest nonlocality argument for CV systems with minimal experimental settings. We then demonstrate the quantum violations of the CV entropic Bell inequality for TMSV states, although a previous belief claims no violation of any Bell inequality in its ordinary form for the same TMSV states using CV quadrature measurements. The parameters required for the violations are well within the experimentally accessible regime. By taking the full merits of the CV entangled light fields, our argument thus opens up a strong possibility for a loophole-free test of local realism.

\section{Acknowledgments}

We thank Wen-Fei Cao for his help in numerical calculations. This work was supported by the National Natural Science Foundation of China under Grant No. 61125502, the Chinese Academy of Sciences, the National High Technology Research and Development Program of China, and the National Fundamental Research Program under Grant No. 2011CB921300.
[1] A. Einstein, B. Podolsky, and N. Rosen, Phys. Rev. 47, 777 (1935).

[2] J.S. Bell, Physics (Long Island) 1, 195 (1964).

[3] J.F. Clauser, M.A. Horne, A. Shimony, and R.A. Holt, Phys. Rev. Lett. 23, 880 (1969).

[4] J.-W. Pan, Z.-B. Chen, C.-Y. Lu, H. Weinfurter, A. Zeilinger, and M. Żukowski, Rev. Mod. Phys. 84, 777 (2012), and references therein.

[5] M.D. Reid and P.D. Drummond, Phys. Rev. Lett. 60, 2731 (1988); M.D. Reid, Phys. Rev. A 40, 913 (1989).

[6] Z.Y. Ou, S.F. Pereira, H.J. Kimble, and K.C. Peng, Phys. Rev. Lett. 683663 (1992).

[7] Z.Y. Ou, S.F. Pereira, and H.J. Kimble, Appl. Phys. B: Photophys. Laser Chem. 55, 265 (1992).

[8] S.L. Braunstein and P. van Loock, Rev. Mod. Phys. 77,
$513(2005)$.

[9] M.D. Reid, P.D. Drummond, W.P. Bowen, E.G. Cavalcanti, P.K. Lam, H.A. Bachor, U.L. Andersen, and G. Leuchs, Rev. Mod. Phys. 81, 1727 (2009).

[10] P. Grangier, M.J. Potasek, and B. Yurke, Phys. Rev. A 38, 3132 (1988).

[11] K. Banaszek and K. Wódkiewicz, Phys. Rev. A 58, 4345 (1998); Phys. Rev. Lett. 82, 2009 (1999).

[12] A. Kuzmich, I.A. Walmsley, and L. Mandel, Phys. Rev. Lett. 85, 1349 (2000).

[13] Z.-B. Chen, J.-W. Pan, G. Hou, and Y.-D. Zhang, Phys. Rev. Lett. 88, 040406 (2002); A.F. Abouraddy, T. Yarnall, B.E.A. Saleh, and M.C. Teich, Phys. Rev. A 75, 052114 (2007); T. Yarnall, A.F. Abouraddy, B.E.A. Saleh, and M.C. Teich, Phys. Rev. Lett. 99, 170408 
(2007).

[14] A. Gilchrist, P. Deuar, and M.D. Reid, Phys. Rev. Lett. 80, 3169 (1998).

[15] H. Nha and H.J. Carmichael, Phys. Rev. Lett. 93, 020401 (2004).

[16] R. García-Patrón, J. Fiurášek, N.J. Cerf, J. Wenger, R. Tualle-Brouri, and Ph. Grangier, Phys. Rev. Lett. 93, 130409 (2004); R. García-Patrón, J. Fiurášek, and N.J. Cerf, Phys. Rev. A 71, 022105 (2005).

[17] M. Paternostro, H. Jeong, and T.C. Ralph, Phys. Rev. A 79, 012101 (2009).

[18] E.G. Cavalcanti, C.J. Foster, M.D. Reid, and P.D. Drummond, Phys. Rev. Lett. 99, 210405 (2007); A. Salles, D. Cavalcanti, and A. Acín, Phys. Rev. Lett. 101, 040404 (2008); Q.Y. He, E.G. Cavalcanti, M.D. Reid, and P.D. Drummond, Phys. Rev. Lett. 103, 180402 (2009).

[19] J.S. Bell, Speakable and Unspeakable in Quantum Mechanics (Cambridge University Press, Cambridge, 1987), Chap. 21.

[20] S.L. Braunstein and C.M. Caves, Phys. Rev. Lett. 61, 662 (1988); Ann. Phys. (NY) 202, 22 (1990).

[21] B.W. Schumacher, Phys. Rev. A 44, 7047 (1991).

[22] N.J. Cerf and C. Adami, Phys. Rev. A 55, 3371 (1997).

[23] T.M. Cover and J.A. Thomas, Elements of Information Theory (John Wiley \& Sons, Inc., Hoboken, New Jersey, 2006).

[24] Ł. Rudnicki, S.P. Walborn, and F. Toscano, Phys. Rev. A 85, 042115 (2012).
[25] J. Schneeloch, P. B. Dixon, G.A. Howland, C.J. Broadbent, and J.C. Howell, Phys. Rev. Lett. 110, 130407 (2013); D.S. Tasca, L. Rudnicki, R.M. Gomes, F. Toscano, and S.P. Walborn, Phys. Rev. Lett. 110, 210502 (2013).

[26] T.C. Zhang, K.W. Goh, C.W. Chou, P. Lodahl, and H.J. Kimble, Phys. Rev. A 67, 033802 (2003); S. Suzuki, H. Yonezawa, F. Kannari, M. Sasaki, and A. Furusawa, Appl. Phys. Lett. 89, 061116 (2006).

[27] S.M. Barnett and P.M. Radmore, Methods in Theoretical Quantum Optics (Oxford Uniersity Press, Oxford, 1997).

[28] G.E. Andrews, R. Askey, and R. Roy, Special Functions (Cambridge University Press, Cambridge, 2000).

[29] For the experimental measurement of quadrature operator's probability distribution for a single-mode light field, see D.T. Smithey, M. Beck, M.G. Raymer, and A. Faridani, Phys. Rev. Lett. 70, 1244 (1993).

[30] A. Aspect, Nature (London) 398, 189 (1999).

[31] M.A. Rowe, D. Kielpinski, V. Meyer, C.A. Sackett, W. M. Itano, C. Monroe, and D.J. Wineland, Nature (London) 409, 791 (2001).

[32] A. Aspect, J. Dalibard, and G. Roger, Phys. Rev. Lett. 49, 1804 (1982).

[33] G. Weihs, T. Jennewein, C. Simon, H. Weinfurter, and A. Zeilinger, Phys. Rev. Lett. 81, 5039 (1998).

[34] O. Aytür and P. Kumar, Phys. Rev. Lett. 65, 1551 (1990). 